\title{
First-principles calculations of dynamic permeability in porous media
}

\author{
Min-Yao Zhou* and Ping Sheng \\ Exxon Research and Engineering Company, Route 22 East, Clinton Township, Annandale, New Jersey 08801
}

(Received 28 November 1988)

\begin{abstract}
By starting from the linearized Navier-Stokes equation for the fluid and the elastic wave equation for the solid frame of a porous medium, the first-principles definition of the frequency-dependent permeability $\kappa(\omega)$ and the recipe for its calculation are derived through the application of the homogenization procedure. It is shown systematically that, in the limit of wavelength much larger than the typical pore size, the fluid may be regarded as incompressible in the permeability calculation, and the solid-frame displacement acts as an additional pressure source term for the fluid flow. The physics underlying the generic asymptotic frequency dependence of $\kappa(\omega)$ is introduced through its analytic solution in the case of a cylindrical tube. To calculate $\kappa(\omega)$ for periodic porous media models, we have formulated a finite-element approach for the numerical solution of the incompressible fluid equations at low and intermediate frequencies and of the Laplace equation at high frequencies. The numerical results for the sinusoidally modulated tube, the fused-spherical-bead lattice, and the fused-diamond lattice indicate a large range of values for the static permeability $\kappa_{0}$ as well as the other asymptotic parameters such as the tortuosity $\alpha$ and the surface length parameter $\Lambda$ as defined by Johnson et al. However, despite their variability, almost all the numerical data on periodic models are shown to satisfy the approximate scaling relation $\kappa(\omega) \cong \kappa_{0} f\left(\omega / \omega_{0}\right)$, where $\omega_{0}$ is a characteristic frequency particular to the medium, and $f$ is a universal function independent of microstructures. We advance arguments that delineate the physical reason for this scaling behavior as well as the condition for its validity. The scaling prediction is then generalized to the case of random porous media through both numerical simulations and the critical-path argument. Our theory gives a simple explanation to the observed correlations in sedimentary rocks, and the scaling prediction is supported by experimental $\kappa(\omega)$ measurements on fused glass beads and crushed-glass samples.
\end{abstract}

\section{INTRODUCTION}

Permeability is a general property of porous media. Its importance in diverse areas of petroleum and chemical industries, ranging from oil production from sedimentary rocks to reaction in zeolites, has long been recognized. In recent years, the study of permeability, especially its relation to the microstructure of a porous medium, was the focus of much theoretical and experimental attention. Among the various results of these studies, it was found that (1) the product of the static permeability $\kappa_{0}$ and the electrical formation factor is directly proportional to a "throat area" measurable by mercury intrusion experiments, ${ }^{1,2}(2)$ in the high-frequency limit the permeability is predicted to contain information about the volume-tosurface ratio of a porous medium,,$^{3,4}$ and (3) a particular combination of high- and low-frequency permeability parameters was noted to be nearly constant for a variety of sedimentary rocks as well as laboratory-produced porous-medium samples. ${ }^{3}$

These findings clearly suggest that there exist some generic relationships between the microstructure and the dynamic permeability function $\kappa(\omega)$, defined phenomenologically as $\mathbf{U}(\omega)=-[\kappa(\omega) / \eta] \nabla p(\omega)$, where $\mathbf{U}$ is the average flow velocity, $\eta$ is the fluid viscosity, and $p$ the applied pressure. It is the purpose of this work to carry out first-principles calculations of $\kappa(\omega)$ for a variety of model porous media and to deduce from the results of these calculations the geometric content of the function $\kappa(\omega)$. Our finding is surprisingly simple and may be expressed by the general validity of the scaling relation 5,6

$$
\kappa(\omega) / \kappa_{0} \simeq f\left(\omega / \omega_{0}\right)
$$

where $\omega_{0}$ is a characteristic frequency particular to the medium and $f$ is a universal function independent of microstructures. Physical arguments are advanced to clarify the geometric meaning of this scaling behavior as well as to delineate the general conditions for its validity. Besides offering a simple explanation for the observed correlations in sedimentary rocks mentioned above, our scaling relation, Eq. (1), predicts that there are only two independent microstructural parameters obtainable from the measurement of $\kappa(\omega)$. This prediction was indeed verified by experimental results on fused-glass beads. ${ }^{6}$

In what follows, the formulation of our approach is given in Sec. II; calculation of $\kappa(\omega)$ for various model systems and the discussion of results are presented in Sec. III. Section IV demonstrates the scaling aspect of our results and discusses its physical underpinnings. Section $\mathrm{V}$ extends the scaling prediction to random networks of 
permeable elements, and Sec. VI concludes with a discussion of further topics.

\section{FORMULATION}

Consider a fluid-filled porous medium with bicontinuous solid and fluid networks under the excitation of an external harmonic source with frequency $\omega$. Since the motions of solid and fluid are coupled by the usual hydrodynamic boundary conditions at pore surfaces, any excitation of one component would inevitably be transmitted to the other component as well. Therefore, the complete problem necessarily involves the joint motion of both the fluid and the solid components in which the local fluid velocity $\mathbf{v}$ is governed by the linearized Navier-Stokes equation

$$
\begin{aligned}
& -i \omega \rho_{f} \mathbf{v}=\nabla \cdot \overleftrightarrow{\sigma}, \\
& \overleftrightarrow{\sigma}=-p \overleftrightarrow{\mathbf{I}}+2 \eta D \nabla \mathbf{v}, \\
& i \omega p=K \nabla \cdot \mathbf{v},
\end{aligned}
$$

where $\rho_{f}$ is the fluid density, $\overleftrightarrow{\sigma}$ denotes the fluid stress tensor, $p$ the fluid pressure, $\eta$ the fluid viscosity, $K$ the fluid bulk modulus, and

$$
[D \nabla \mathbf{v}]_{i j}=\frac{1}{2}\left(\partial_{i} v_{j}+\partial_{j} v_{i}-\frac{2}{3} \delta_{i j} \nabla \cdot \mathbf{v}\right) .
$$

The displacement $\mathbf{u}$ of the solid frame, on the other hand, satisfies the elastic wave equation

$$
\begin{aligned}
& -\omega^{2} \rho_{s} \mathbf{u}=\nabla \cdot \overleftrightarrow{\tau}, \\
& \overleftrightarrow{\tau}=\underline{C} \nabla \mathbf{u},
\end{aligned}
$$

where $\rho_{s}$ is the solid density, $\overleftrightarrow{\tau}$ is the solid stress tensor, and $\underline{C}$ is the fourth-rank elastic tensor. The variables $\mathbf{u}$ and $\mathbf{v}$ are coupled at the fluid-solid interface by the boundary conditions for the continuity of displacement,

$$
\mathbf{v}=-i \omega \mathbf{u},
$$

and the continuity of traction,

$$
\mathbf{n} \cdot \overleftrightarrow{\sigma}=\mathbf{n} \cdot \overleftrightarrow{\tau}
$$

where $\mathbf{n}$ is the unit normal of the fluid-solid interface.

The general solution of Eqs. (2a)-( $2 \mathrm{~g}$ ) for any nontrivial pore geometry would be difficult, if not impossible. However, in actual cases of interest one may almost always identify a dimensionless small parameter $\epsilon$ in the problem that would enable significant simplifications for the permeability calculation. This small parameter is the ratio between two length scales in which the small scale $a$ is given by the typical pore size, and the large scale $L$ is given by the product of the fluid sound speed $c_{0}=\sqrt{K / \rho_{f}}$ and an intrinsic viscous relaxation time $\tau=\rho f a^{2} / \eta$, i.e., $\epsilon=a / c_{0} \tau$. Here $\tau$ has the physical significance as the time scale at which the inertial force density inside a pore, $\rho a / \tau^{2}$, equals the viscous force density, $\eta / \tau a$. It follows that for $\omega>2 \pi / \tau$ the inertial effect dominates, and vice versa for $\omega<2 \pi / \tau$. For water in sedimentary rocks with $a \simeq 1 \mu \mathrm{m}, \epsilon$ is on the order of $10^{-3}$.

By expressing time in units of $\tau$ and length in units of $a$, Eqs. (2a) $-(2 \mathrm{~g})$ may be nondimensionalized as

$$
\begin{aligned}
& -i \omega \mathbf{v}=\epsilon^{-1} \nabla \cdot \overleftrightarrow{\sigma} \text { in } D_{f}, \\
& \overleftrightarrow{\sigma}=-p \overleftrightarrow{\mathbf{I}}+\epsilon 2 D \nabla \mathbf{v} \text { in } D_{f}, \\
& i \omega p=\epsilon^{-1} \nabla \cdot \mathbf{v} \text { in } D_{f}, \\
& -\rho \omega^{2} \mathbf{u}=\epsilon^{-1} \nabla \cdot \overleftrightarrow{\tau} \text { in } D_{s}, \\
& \overleftrightarrow{\tau}=\underline{C} \epsilon^{-1} \nabla \mathbf{u} \text { in } D_{s}, \\
& \mathbf{v}=-i \omega \mathbf{u} \text { on } \partial D_{f}=\partial D_{s}, \\
& \mathbf{n} \cdot \overleftrightarrow{\sigma}=\mathbf{n} \cdot \overleftrightarrow{\tau} \text { on } \partial D_{f}=\partial D_{s}
\end{aligned}
$$

Here $\rho=\rho_{s} / \rho_{f}, \underline{C}$ is in units of $K$, and $p, \overleftrightarrow{\sigma}$, and $\overleftrightarrow{\tau}$ are in units of $L \eta^{2} / \rho_{f} a^{3}$ following the assumption that the macroscopic pressure gradient should be on the same order as $\eta v / a^{2}$ in accordance with Darcy's law. This last assumption will be justified by our consistent derivation of Darcy's law from Eqs. (3a)-(3g).

The existence of a small parameter in Eq. (3) means that we can apply the well-known technique of homogenization $^{7}$ that would enable us to derive not only the equations governing the macroscopically measurable quantities (such as the average fluid velocity $U$ ), but also the recipes for calculating the phenomenological parameters (such as the permeability $\kappa$ ) from microstructures. The technique basically consists of three steps. First, the existence of two length scales in the problem is explicitly recognized by writing all the field variables $\mathbf{v}, \mathbf{u}, \overleftrightarrow{\sigma}, \overleftrightarrow{\tau}$, and $p$ as a function of $\epsilon$ plus the two spatial scales $\mathrm{x}=\mathrm{r} / L$ and $\mathbf{y}=\mathbf{r} / a$. The field variables are then expanded as a power series in $\epsilon$. For example,

$$
\mathbf{v}_{\epsilon}(\mathbf{x}, \mathbf{y})=\mathbf{v}_{0}(\mathbf{x}, \mathbf{y})+\epsilon \mathbf{v}_{1}(\mathbf{x}, \mathbf{y})+\epsilon^{2} \mathbf{v}_{2}(\mathbf{x}, \mathbf{y})+\cdots .
$$

Second, the gradient operation $\nabla$ is divided into two parts, one acting on the $\mathbf{x}$ scale and one on the $\mathbf{y}$ scale:

$$
\nabla \rightarrow \epsilon \nabla_{x}+\nabla_{y} .
$$

Third, by equating terms with the same power of $\epsilon$, we get a hierarchy of equations for the quantities $\mathbf{v}_{0}, \mathbf{v}_{1}, \mathbf{u}_{0}$, $\mathbf{u}_{1}, \overleftrightarrow{\sigma}_{0}, \overleftrightarrow{\sigma}_{1}, p_{0}, p_{1}$, etc. For our purpose the relevant lowest-order $\left(\epsilon^{-1}\right)$ equations are

$$
\begin{aligned}
& \nabla_{y} \cdot \overleftrightarrow{\sigma}_{0}=0, \\
& \overleftrightarrow{\sigma}_{0}=-p_{0} \stackrel{\mathbf{I}}{ } \\
& \nabla_{y} \cdot \mathbf{v}_{0}=0 \\
& \nabla_{y} \mathbf{u}_{0}=0 .
\end{aligned}
$$

Equation (6d) tells us that $\mathbf{u}_{0}=\mathbf{u}_{0}(\mathbf{x})$, i.e., $\mathbf{u}_{0}$ is a function of $\mathbf{x}$ only. Equation (6b) shows that $\overleftrightarrow{\sigma}_{0}$ is proportional to an identity matrix (where $p_{0}$ may be regarded as the externally applied pressure), and when this information is combined with Eq. (6a), we conclude that $\overleftrightarrow{\sigma}_{0}=\overleftrightarrow{\sigma}(\mathbf{x})$ also. Next, Eq. (6c) indicates that as far as the fluid is concerned, to the lowest order it may be regarded as incompressible on the $y$ scale. This is physically reasonable since for $\omega \ll<\epsilon^{-1}$, the acoustic wavelength $\lambda \gg a$, which implies compressibility effect is on the order of 
$a / \lambda \sim \epsilon \ll 1$. The relevant boundary condition relating $\mathbf{u}_{0}$ and $\mathbf{v}_{0}$ is given by

$$
\mathbf{v}_{0}=-i \omega \mathbf{u}_{0} \text {. }
$$

Before writing down the next order equations we would like to follow Biot, ${ }^{8}$ and Burridge and Keller, ${ }^{7}$ in expressing $\mathbf{v}_{0}$ in terms of $\mathbf{u}_{0}$ and a relative fluid displacement $\mathbf{w}$

$$
\mathbf{v}_{0}=-i \omega\left[\mathbf{u}_{0}(\mathbf{x})+\mathbf{w}(\mathbf{x}, \mathbf{y})\right],
$$

Since $\nabla_{y} \cdot \mathbf{u}_{0}(\mathbf{x})=0$, the condition $\nabla_{y} \cdot \mathbf{v}_{0}$ directly translates into

$$
\boldsymbol{\nabla}_{y} \cdot \mathbf{w}=0,
$$

with the simple boundary condition

$$
\mathbf{w}=0 \text { on } \partial D_{f}=\partial D_{s} \text {. }
$$

In terms of $w$, the $\epsilon^{0}$ order equations from Eqs. (3a) and (3b) are

$$
\begin{aligned}
& \nabla_{y} \cdot \overleftrightarrow{\sigma}_{1}+\omega^{2} \mathbf{w}=-\left(\nabla_{x} \cdot \overleftrightarrow{\sigma}_{0}+\omega^{2} \mathbf{u}_{0}\right) \\
& \overleftrightarrow{\sigma}_{1}=-p_{1} \overleftrightarrow{\mathbf{I}}-i \omega D \nabla_{y} \mathbf{w} .
\end{aligned}
$$

By substituting Eq. (9b) into (9a), we get

$$
-\nabla_{y} p_{1}-i \omega \nabla_{y}^{2} \mathbf{w}+\omega^{2} \mathbf{w}=-\left(-\nabla_{x} p_{0}+\omega^{2} \mathbf{u}_{0}\right) .
$$

Since $p_{1}$ and $\mathbf{w}$ are solutions to a set of linear equations, they may be formally expressed as linear operators acting on the source term $\left(-\nabla_{x} p_{0}+\omega_{2} \mathbf{u}_{0}\right)$, i.e.,

$$
\begin{aligned}
& p_{1}=\mathbf{P}(\mathbf{x}, \mathbf{y}) \cdot\left(-\nabla_{x} p_{0}+\omega^{2} \mathbf{u}_{0}\right) \\
& \mathbf{w}=\overleftrightarrow{\mathbf{w}}(\mathbf{x}, \mathbf{y}) \cdot\left(-\nabla_{x} p_{0}+\omega^{2} \mathbf{u}_{0}\right)
\end{aligned}
$$

By substituting Eqs. (10a) and (10b) into Eqs. (9c) and (8a), we get the generic equations satisfied by the operators $\mathbf{P}$ and $\overleftrightarrow{\mathbf{W}}$

$$
\begin{aligned}
& -\nabla_{y} \mathbf{P}-i \omega \nabla_{y}^{2} \overleftrightarrow{\mathrm{W}}+\omega^{2} \overleftrightarrow{\mathrm{W}}=-\overleftrightarrow{\mathrm{I}}, \\
& \nabla_{y} \cdot \overleftrightarrow{\mathrm{W}}=0,
\end{aligned}
$$

with $\overleftrightarrow{\mathbf{W}}=0$ at the fluid-solid interfaces. Given the solution $\overleftrightarrow{\mathrm{W}}$, the dynamic permeability $\kappa(\omega)$ may be directly calculated by noting that the average flow rate $\mathbf{U}$ can be obtained from Eq. (10b) by averaging $\mathbf{w}$ over the pore scale y

$$
\mathbf{U}=-i \omega\langle\mathbf{w}\rangle_{y}=-i \omega\langle\overleftrightarrow{\mathrm{W}}(\mathbf{x}, \mathbf{y})\rangle_{y} \cdot\left(-\nabla_{x} p_{0}+\omega^{2} \mathbf{u}_{0}\right) .
$$

Equation (12) may be regarded as the generalized Darcy's law for elastic porous media since it tells us that the elastic solid displacement acts as an additional excitation source in the form of $\omega^{2} \mathbf{u}_{0}$. Comparing Eq. (12) with the usual form of Darcy's law, we get

$$
\overleftrightarrow{\kappa}(\omega)=-i \omega\langle\overleftrightarrow{\mathbf{W}}(\mathbf{x}, \mathbf{y})\rangle_{y}
$$

as the definition of the dynamic permeability tensor. $\overleftrightarrow{\kappa}(\omega)$ is expected to reduce to a scalar in the case of unidirectional, isotropic, or simple-cubic microstructures.
To summarize, the homogenization procedure has given us the following three results. First, it shows that the fluid may be regarded as incompressible on the pore scale. Second, it shows that the solid frame displacement may be regarded as a source term and therefore does not enter the generic problem of permeability calculation. Third, it justifies Darcy's law from first principles. We should note here that in the above, only part of the starting equations have been examined under the homogenization procedure. Consideration of all the equations would lead to Biot's equations as shown by Burridge and Keller. ${ }^{7}$

\section{PERMEABILITY CALCULATION}

\section{A. Analytic solution for a cylindrical tube}

The simplest case of permeability calculation would be that for a cylindrical tube with radius 1 . The examination of its analytic solution ${ }^{9}$ can yield a physical understanding of the velocity profile as a function of frequency and the generic asymptotic frequency dependence of $\kappa(\omega)$ that follows. For a tube, Eqs. (11a) and (11b) reduce to a vector equation with $\overleftrightarrow{\mathrm{I}} \rightarrow \widehat{\mathbf{e}}_{z}$. From symmetry, we expect $\overleftrightarrow{\mathbf{W}}$ to be diagonal with $W_{z z}=w$ and $\mathbf{P}=p_{z} \widehat{\mathbf{e}}_{z}$ as the only nonzero components. That means $\partial p_{z} / \partial r=0$, or $p_{z}=p_{z}(z)$. From translational invariance we also expect $\partial p_{z} / \partial z=$ constant, and the constant can be taken to be zero if we regard $\partial p_{z} / d z$ as that part of the pressure gradient which represents the correction to the (constant) applied pressure gradient. Also, the incompressibility condition becomes $\partial w / \partial z=0$, or $w=w(r)$. Equation (11a) can therefore be written as (with subscript $y$ dropped),

$$
r^{2} \frac{\partial^{2} w}{\partial r^{2}}+r \frac{\partial w}{\partial r}+i \omega r^{2}\left(w+\frac{1}{\omega^{2}}\right)=0 .
$$

By making the transformation of variables

$$
\begin{aligned}
& r^{\prime}=\sqrt{i \omega} r, \\
& w^{\prime}=w+\frac{1}{\omega^{2}},
\end{aligned}
$$

we get

$$
\left(r^{\prime}\right)^{2} \frac{\partial^{2} w^{\prime}}{\partial\left(r^{\prime}\right)^{2}}+r^{\prime} \frac{\partial w^{\prime}}{\partial r^{\prime}}+\left(r^{\prime}\right)^{2} w^{\prime}=0
$$

This is the Bessel equation of order zero. Since $w$ has to be regular at $r=0$, we get

$$
w^{\prime}=c J_{0}\left(r^{\prime}\right)=c J_{0}(\sqrt{i \omega} r),
$$

or

$$
w=w^{\prime}-\frac{1}{\omega^{2}}=c J_{0}(\sqrt{i \omega} r)-\frac{1}{\omega^{2}} .
$$

The requirement of $w=0$ at $r=1$ means that

$$
c=\frac{1}{\omega^{2}}\left[J_{0}(\sqrt{i \omega})\right]^{-1},
$$

so the final solution is given by 


$$
w=-\frac{1}{\omega^{2}}\left(1-\frac{J_{0}(\sqrt{i \omega} r)}{J_{0}(\sqrt{i \omega})}\right) .
$$

From Eq. (13), the dynamic permeability is given by

$$
\begin{aligned}
\kappa(\omega) & =\frac{i}{\pi \omega} \int_{0}^{1} 2 \pi r d r\left[1-\frac{J_{0}(\sqrt{i \omega} r)}{J_{0}(\sqrt{i \omega})}\right] \\
& =\frac{i}{\omega}\left[1-\frac{2}{\sqrt{i \omega}} \frac{J_{1}(\sqrt{i \omega})}{J_{0}(\sqrt{i \omega})}\right] .
\end{aligned}
$$

For a tube of arbitrary radius $a$, Eq. (17) still applies provided that $\kappa(\omega)$ be measured in units of $a^{2}$ and $\omega$ be measured in units of $\eta / \rho_{f} a^{2}$.

A side result of the analytic $\kappa(\omega)$ solution is the velocity profile in a tube, $-i \omega w$, where $w$ is given by Eq. (16b). A plot of the real and imaginary parts of the velocity for four different frequencies is given in Figs. 1(a)-(1d). At low frequencies, the real part has a parabolic profile as expected. The small imaginary part, which represents the out-of-phase inertial effect, increases linearly with frequency because it represents acceleration. At $\omega_{c} \simeq 5$, the real and imaginary parts cross in magnitude and for $\omega>\omega_{c}$ the imaginary part always dominates. The $\omega_{c}$ therefore represents the frequency at which the inertial effect overtakes the viscous effect. The profile of the imaginary part at high frequencies, as seen in Figs. 1(c) and $1(d)$, is described by a rather flat central region with a small maximum and then a steep decline close to the wall. The flat central region may be identified as the zone for wave propagation. On the macroscopic spatial scale, its amplitude is modulated sinusoidally. The maximum amplitude is seen to decrease linearly with frequency because for a given force, displacement $w \sim \omega^{-2}$ so that $v=-i \omega w \sim i \omega^{-1}$. The real part, on the other hand, is nearly zero everywhere except for a layer near the wall. The thickness of this boundary layer represents the viscous relaxation length $l=\sqrt{2 \eta / \rho_{f} \omega}$. The $\omega^{-1 / 2}$ fre-
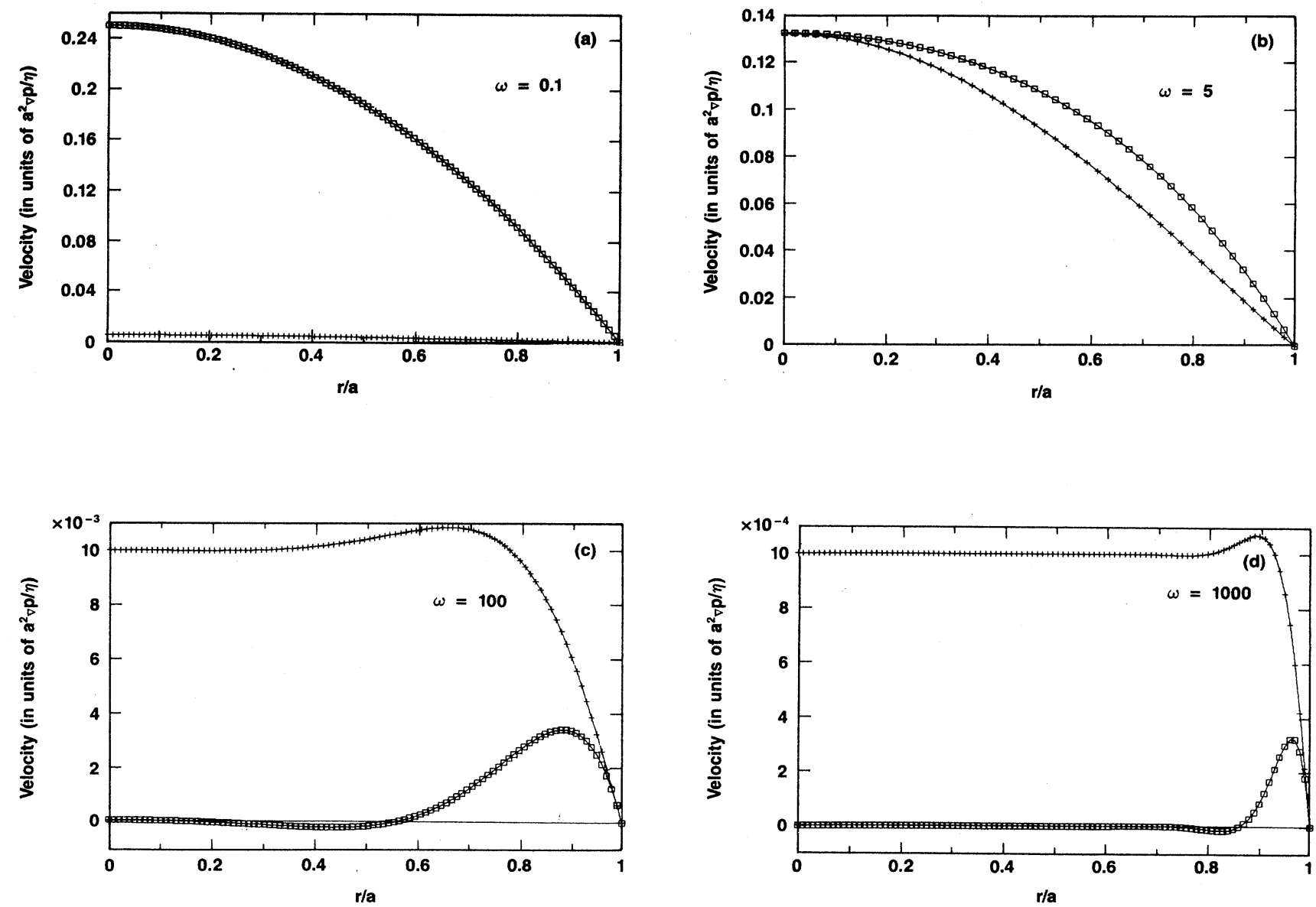

FIG. 1. Fluid-velocity profile in a cylindrical tube at four different frequencies: (a) $\omega=0.1$, (b) $\omega=5$, (c) $\omega=100$, (d) $\omega=1000$. Frequency is expressed in units of $\eta / \rho_{f} a$. The square denotes the real (in-phase with applied pressure) part of the velocity, and the cross denotes the imaginary (out-of-phase) part of the velocity. 
quency dependence may be easily verified by visual inspection of Figs. 1(c) and 1(d). The maximum of the real part in the boundary layer is seen to decrease as $\omega^{-1}$, and the average of the real part should therefore have a fre- quency dependence of $\omega^{-1 / 2} \cdot \omega^{-1}=\omega^{-3 / 2}$. Since the permeability is nothing more than the average velocity, the above discussion implies the following asymptotic frequency dependence in restored units

$$
\kappa(\omega)=\left\{\begin{array}{l}
A S+i B S^{2}\left(\rho_{f} / \eta\right) \omega, \quad \omega \rightarrow 0, \\
C S^{-1 / 2}\left(\eta / \rho_{f}\right)^{3 / 2} \omega^{-3 / 2}+i D\left(\eta / \rho_{f}\right) \omega^{-1}, \omega \rightarrow \infty
\end{array}\right.
$$

where $A, B, C, D$ are dimensionless constants and $S$ denotes the cross-sectional area. Indeed, the asymptotic expansion of Eq. (17) verifies this form with the values of $A=(8 \pi)^{-1}, B=\left(48 \pi^{2}\right)^{-1}, C=(2 \pi)^{1 / 2}$, and $D=1$. For straight tubes of noncircular cross sections, dimensional analysis tells us that only the shape constants $A, B, C$, and $D$ are changed in Eq. (18). The calculation of these shape constants has been discussed by Norris. ${ }^{10}$

\section{B. Numerical solutions for periodic models}

To calculate $\kappa(\omega)$ for periodic porous media with arbitrary unit-cell microstructures, we have to solve Eq. (11) numerically. This is accomplished by formulating a finite-element approach as follows. Consider an action functional with the following form:

$$
\begin{gathered}
G=\int d^{3} r\left\{\frac{i}{2} \omega\left[\left(\frac{\partial \mathbf{w}_{z}}{\partial x}\right)^{2}+\left(\frac{\partial \mathbf{w}_{z}}{\partial y}\right)^{2}+\left(\frac{\partial \mathbf{w}_{z}}{\partial z}\right)^{2}\right]\right. \\
\left.+\frac{1}{2} \omega^{2} \mathbf{w}_{z} \cdot \mathbf{w}_{z}+\left(\widehat{\mathbf{e}}_{z}-\nabla p_{z}\right) \cdot \mathbf{w}_{z}\right\},
\end{gathered}
$$

where $z$ is the direction of the applied pressure, $\mathbf{w}_{z}$ denotes the row vector $\left(W_{z x}, W_{z y}, W_{z z}\right)$ in the $\overleftrightarrow{\mathrm{W}}$ matrix, $p_{z}$ is the $z$ component of the $\mathbf{P}$ vector, $\widehat{\mathbf{e}}_{z}$ the unit vector in the $z$ direction, and the integration is over the pore space in a unit cell. By dropping the $z$ subscript and setting the functional variation $\delta G$ to zero, we get

$$
\begin{aligned}
\delta G= & \int d^{3} r\left(-i \omega \nabla^{2} \mathbf{w}+\omega^{2} \mathbf{w}+\widehat{\mathbf{e}}-\nabla p\right) \cdot \delta \mathbf{w} \\
& +\int d^{3} r(\nabla \cdot \mathbf{w}) \delta p-\int d \mathbf{S} \cdot \mathbf{w} \delta p \\
& +\int d \mathbf{S} \cdot \nabla \mathbf{w} \cdot \delta \mathbf{w}(i \omega)=0 .
\end{aligned}
$$

The last two terms of $\delta G$ are integrals over the surface of a unit cell, consisting partly of fluid-solid interfaces and partly of fluid-fluid interfaces at the unit cell boundary. The contribution over the fluid-solid interaces is identically zero due to the boundary condition that $w=0$ on $\partial D_{f}=\partial D_{s}$ (therefore $\delta \mathrm{w}=0$ also). For unit-cell surfaces that have fluid-fluid contact, we apply the periodic symmetry to the quantity $\mathbf{w}$ and $\delta p$ and then note that the normals to the surface must be opposite to each other at two ends of the cell. That means the surface contributions are identically zero, yielding

$$
\begin{aligned}
& -\nabla p-i \omega \nabla^{2} \mathbf{w}+\omega^{2} \mathbf{w}=-\widehat{\mathbf{e}}, \\
& \boldsymbol{\nabla} \cdot \mathbf{w}=0,
\end{aligned}
$$

which are exactly the equations to be satisfied by $\mathbf{w}$ and $p$.
By discretizing the pore space into tetrahedron elements and associating four variables $p_{\beta}, w_{x \beta}, w_{y \beta}, w_{z \beta}$ with each node $\beta$, the action $A$ may be approximated by a finite sum. Setting the derivatives $\partial G / \partial w_{i \beta}=0$, $\partial G / \partial p_{\beta}=0$ then results in a set of linear simultaneous equations which may be arranged in a band format so that we can apply the fast band-matrix solver. ${ }^{11}$ It should be noted here that the finite-element approach is especially well suited to this problem because the flexibility of the discretization scheme makes it easy to adapt to the odd geometry of the pore space as well as to the special requirement that the behavior near the wall must be well resolved so as to capture the effects of the boundary layer. However, since the boundary-layer thickness decreases as frequency increases, at very high frequencies the solution accuracy inevitably suffers. To overcome this problem, we follow the work of Johnson et al. ${ }^{3}$ in noting that the viscous term $i \omega \nabla^{2} w$ in the Navier-Stokes equation is negligible compared to the inertial term $\omega^{2} \mathbf{w}$ at high frequencies (except near the wall). Therefore, for the bulk of the fluid one can write

$$
\begin{aligned}
& -\boldsymbol{\nabla} p+\omega^{2} \mathbf{w}=-\widehat{\mathbf{e}}, \\
& \boldsymbol{\nabla} \cdot \mathbf{w}=0 .
\end{aligned}
$$

The boundary conditions on $\mathbf{w}$ consist of $\mathbf{w} \cdot \mathbf{n}=0$ at $\partial D_{f}=\partial D_{s}$, where $\mathbf{n}$ is the surface normal, and the requirement that $\mathbf{w}$ be periodic at fluid-fluid cell interfaces. By taking the divergence of Eq. (21a) and using Eq. (21b), we get a Laplace equation for $p$,

$$
\nabla^{2} p=0
$$

with the boundary conditions of $(\nabla p-\widehat{\mathbf{e}}) \cdot \mathbf{n}=0$ at $\partial D_{f}=\partial D_{s}$ and $\nabla p-\widehat{\mathbf{e}}$ being periodic at fluid-fluid cell interfaces as required by Eq. (21a) and the condition on $\mathbf{w}$. To obtain accurate solutions at high frequencies, we therefore use the finite-element approach to solve Eq. (22) in the pore space, with the action given by

$$
G=\frac{1}{2} \int d^{3} r \nabla p \cdot \nabla p
$$

Once the solution of $p$ is obtained (up to a constant, which may be fixed by letting the pressure value at a particular boundary node equal to 0 ), the value of $w$ is obtained as

$$
\mathbf{w}=\frac{1}{\omega^{2}}(\nabla p-\widehat{\mathbf{e}})=\frac{1}{\omega^{2}} \nabla p_{t}
$$

where we denote the total pressure gradient, the sum of applied and flow-induced gradients, as $\nabla p_{t}$. The value of 
w obtained in this fashion is always real, which means that

$$
\begin{aligned}
\lim _{\omega \rightarrow \infty}[\operatorname{Im} \kappa(\omega)] & =\omega\langle\mathbf{w} \cdot \hat{\mathbf{e}}\rangle \\
& =\omega^{-1} \frac{\int \nabla p_{t} \cdot \hat{\mathbf{e}} d^{3} r}{V}=\phi \alpha^{-1} \omega^{-1},
\end{aligned}
$$

where $V$ is the unit cell volume and we have assumed that $\langle\mathbf{w}\rangle$ is in the direction of applied pressure $\widehat{\mathbf{e}}$ (which must be true for pore geometry that is isotropic, simple cubic, or uniaxial), $\phi$ denotes the porosity, and $\alpha$ is defined as the tortuosity factor that has the following physical meaning. On the macroscopic scale, we have the equation

$$
i \omega p=-K \nabla \cdot\langle i \omega \mathbf{w}\rangle=-K \kappa(\omega) \nabla^{2} p / \eta .
$$

Since the imaginary part of $\kappa(\omega)$ dominates at high frequencies, we write $\kappa(\omega)=i \alpha^{-1} \eta / \omega \rho_{f}$. This yields a wave equation

$$
\omega^{2} p+\left[\left(K / \rho_{f}\right) / \alpha\right] \nabla^{2} p=0
$$

in which the sound speed is given by $\sqrt{K / \rho_{f}} / \sqrt{\alpha}$ $=c_{0} / \sqrt{\alpha}$. One way to interpret this result is that the effective distance of travel for the wave between two points is increased by $\sqrt{\alpha}$ due to the tortuosity of the path.

The $\alpha$ factor is easily related to the electrical conductivity $\sigma$ of a porous medium. That is, since $\sigma$ is given by the average of the electrical current density divided by the unit applied field, we have

$$
\sigma=\frac{\int \sigma_{0} \nabla \Phi \cdot \widehat{\mathbf{e}} d^{3} r}{V}=\sigma_{0} F^{-1},
$$

where $\Phi$ is the electrical potential, $\sigma_{0}$ the conductivity of the pore fluid, and $F$ is denoted as the formation factor that comprises the geometric information measured by electrical conductivity. Since $\Phi$ satisfies the Laplace equation with the boundary condition that $\boldsymbol{\nabla} \Phi \cdot \mathbf{n}=0$ (no normal current), $\Phi$ is exactly analogous to $p$. Therefore,

$$
F=\phi^{-1} \alpha \text {. }
$$

Equation (27) shows that the limiting behavior of $\operatorname{Im}[\kappa(\omega)]$ can be completely determined by electrical measurement. This fact was first pointed out some time ago by Rayleigh ${ }^{12}$ and Brown. ${ }^{13}$

What about the real part of $\kappa(\omega)$ in the limit of $\omega \rightarrow \infty$ ? Since the real part of $\kappa(\omega)$ arises purely from the boundary layer, to extract its behavior we have to define a local coordinate so as to resolve the variation of $\mathbf{w}$ inside the layer. Because the boundary-layer thickness is small, we can treat the pore surface as locally flat without incurring much error. Let us define $\xi$ as the coordinate normal to the surface, and $\mathbf{w}, \nabla p_{t}=\omega^{2} \mathbf{w}_{0}$ as the displacement and pressure gradient parallel to the surface. Here by $w_{0}$ we denote the value of $\nabla p_{t} / \omega^{2}$ at the boundary obtained from the solution of the Laplace equation. From Eq. (20b), the equation for $w$ is then

$$
\frac{d^{2}}{d \xi^{2}} \mathbf{w}+i \omega \mathbf{w}=i \omega \mathbf{w}_{0},
$$

with the boundary conditions that $\mathbf{w}=0$ at $\xi=0$, and $\mathbf{w}=\mathbf{w}_{0}$ at $\xi=\infty$. The solution is

$$
\mathbf{w}=\mathbf{w}_{0}[1-\exp (-\sqrt{i \omega} \xi)] \text {. }
$$

The real part of $\kappa(\omega)$ is therefore given by

$$
\begin{aligned}
\lim _{\omega \rightarrow \infty}[\operatorname{Re} \kappa(\omega)] & =-\omega \operatorname{Im}\langle\mathbf{w} \cdot \hat{\mathbf{e}}\rangle=\omega\left\langle\operatorname{Im}\left[\left(\mathbf{w}_{0} \cdot \hat{\mathbf{e}}\right) \exp (-\sqrt{i \omega} \xi)\right]\right\rangle \\
& =\operatorname{Im}\left[\frac{\omega}{V} \int_{\partial D_{f}=\partial D_{s}} d S\left(\mathbf{w}_{0} \cdot \hat{\mathbf{e}}\right) \int_{0}^{\infty} d \xi \exp (-\sqrt{i \omega} \xi)\right] \\
& =\operatorname{Im}\left[\frac{\omega}{V} \int_{\partial D_{f}=\partial D_{s}} d S\left(\mathbf{w}_{0} \cdot \hat{\mathbf{e}}\right)(i \omega)^{-1 / 2}\right] \\
& =\frac{1}{\sqrt{2}}\left[\frac{\omega^{1 / 2}}{V} \int_{\partial D_{f}=\partial D_{s}} d S \mathbf{w}_{0} \cdot \hat{\mathbf{e}}\right] \\
& =\frac{1}{\sqrt{2}}\left[\omega^{-3 / 2}\left[\int_{\partial D_{f}=\partial D_{s}} d S \nabla p_{t} \cdot \hat{\mathbf{e}} / \int_{\text {pore }} d^{3} r \nabla p_{t} \cdot \hat{\mathbf{e}}\right] \phi \alpha^{-1}\right],
\end{aligned}
$$

where we have specified the surface integral to be only over the fluid-solid interface where there is a boundary layer, and substituted $\nabla p_{t} / \omega^{2}$ for $w_{0}$ and again assumed that $\langle\mathbf{w}\rangle$ is in the direction of applied pressure. If we define a length parameter $\Lambda$ as

$$
\left.\frac{2}{\Lambda}=\iint_{\partial D_{f}=\partial D_{s}} \nabla p_{t} \cdot \hat{\mathbf{e}} d S / \int_{\text {pore }} \nabla p_{t} \cdot \widehat{\mathbf{e}} d^{3} r\right]
$$

then

$$
\lim _{\omega \rightarrow \infty} \operatorname{Re} \kappa(\omega)=\frac{\sqrt{2} \phi}{\Lambda \alpha} \omega^{-3 / 2} .
$$


The $\Lambda$ parameter and its significance as a weighted measure of volume-to-surface ratio were first pointed out by Johnson et al. ${ }^{3}$ Equations (31) and (32) complete the Laplace-equation formulation for the calculation of $\kappa(\omega)$ in the limit of $\omega \rightarrow \infty$. Since, at low frequencies, the real part of $\kappa(\omega)$ is given by its static value $\kappa_{0}$ and the imaginary part necessarily varies as $\omega$ due to its inertial origin, we can write, in restored units, the general asymptotic behavior ${ }^{4}$ of $\kappa(\omega)$,

$$
\kappa(\omega)=\left\{\begin{array}{l}
\kappa_{0}+i C_{1}\left(\rho_{f} / \eta\right) \omega, \omega \rightarrow 0 \\
{[\sqrt{2} \phi /(\alpha \Lambda)]\left(\eta / \rho_{f}\right)^{3 / 2} \omega^{-3 / 2}+i(\phi / \alpha)\left(\eta / \rho_{f}\right) \omega^{-1}, \omega \rightarrow \infty}
\end{array}\right.
$$

In the above we have outlined the approaches for the numerical evaluation of $\kappa(\omega)$. In Fig. 2 we show the three models on which the numerical calculations have been implemented. The first one is a cylindrical tube with sinusoidal modulation of its cross section, i.e.,

$$
S(z)=\pi a^{2}[1-(\delta / 2)(1-\cos k z)]^{2},
$$

where $a$ is the maximum radius, $\delta$ is a modulation parameter, and $k$ gives the modulation periodicity. The second model is formed by the remains of spheres of radius $a(1+\delta)$ after six caps of height $a \delta$ are cleaved off so that they can be fitted on a simple-cubic lattice with lattice constant $2 a$. The third model is similar to the second one except the spheres are replaced by octahedrons of half- diagonal $a(1+\delta)$ with six caps of height $a \delta$ cleaved off. It is noted that $\delta$ is a parameter that controls the porosity $\phi$ and the microstructure in all three models. In the following figures we give a sampling of results from the finite-element approach for the solution of the fluid equations and the Laplace equation. Due to the symmetries of models II and III, considerable computational saving and accuracy were achieved by solving for $\mathbf{w}$ and $p$ in only one-eighth of the unit cell. The number of nodes and their distribution in the solution region were varied, with attention paid especially to the throat and the nearwall areas, to examine the accuracy and convergence of the solutions. For narrow throats, up to a total of $5 \times 10^{4}$ nodal points were used to guarantee the variation of the

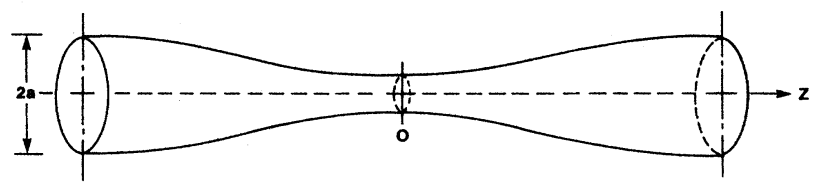

MODEL I
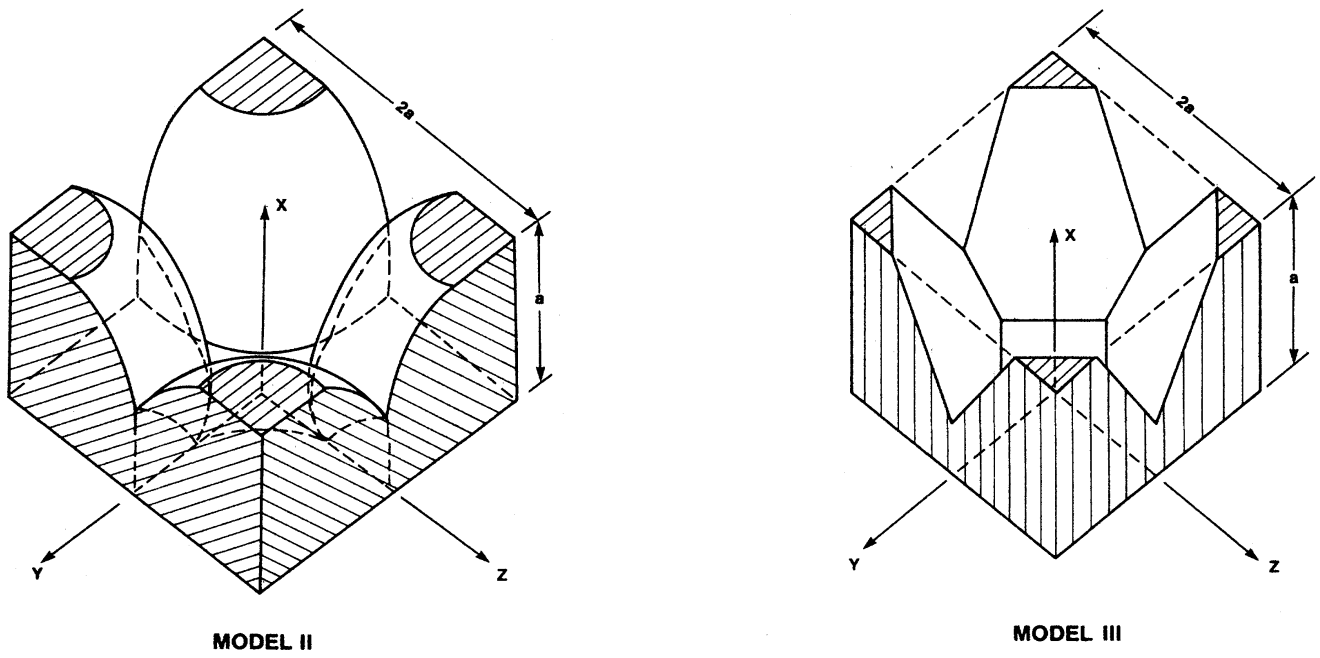

MODEL III

FIG. 2. Cross-sectional view of the pore geometries for the three periodic models. 
TABLE I. List of model parameters, the calculated asymptotic constants $\kappa_{0}, C_{1}, \Lambda$, and $\alpha$, and the values of $F_{1}$ and $F_{2}$ for the three periodic porous-media models.

\begin{tabular}{|c|c|c|c|c|c|c|c|c|}
\hline Model & $\delta$ & $\phi$ & $\kappa_{0} / a^{2} \phi$ & $C_{1} / a^{4}$ & $\alpha$ & $\Lambda / a$ & $\begin{array}{c}F_{1} \\
\alpha \kappa_{0}^{2} \\
C_{1} \phi\end{array}$ & $\begin{array}{c}F_{2} \\
\left.\frac{\Lambda^{2} \phi}{\alpha \kappa_{0}}\right]^{1 / 2}\end{array}$ \\
\hline \multirow[t]{14}{*}{ I } & 0.00 & 1.000 & $0.125 \times 10^{0}$ & $0.208 \times 10^{-1}$ & 1.000 & 1.000 & 0.750 & 2.828 \\
\hline & 0.10 & 0.9050 & $0.111 \times 10^{0}$ & $0.165 \times 10^{-1}$ & 1.007 & 0.947 & 0.752 & 2.832 \\
\hline & 0.20 & 0.8200 & $0.939 \times 10^{-1}$ & $0.121 \times 10^{-1}$ & 1.032 & 0.885 & 0.752 & 2.844 \\
\hline & 0.30 & 0.7450 & $0.748 \times 10^{-1}$ & $0.807 \times 10^{-2}$ & 1.082 & 0.812 & 0.751 & 2.855 \\
\hline & 0.40 & 0.6800 & $0.548 \times 10^{-1}$ & $0.469 \times 10^{-2}$ & 1.173 & 0.729 & 0.751 & 2.877 \\
\hline & 0.50 & 0.6250 & $0.358 \times 10^{-1}$ & $0.227 \times 10^{-2}$ & 1.327 & 0.636 & 0.750 & 2.919 \\
\hline & 0.55 & 0.6012 & $0.273 \times 10^{-1}$ & $0.144 \times 10^{-2}$ & 1.446 & 0.584 & 0.749 & 2.939 \\
\hline & 0.60 & 0.5800 & $0.199 \times 10^{-1}$ & $0.846 \times 10^{-3}$ & 1.608 & 0.529 & 0.753 & 2.957 \\
\hline & 0.65 & 0.5612 & $0.136 \times 10^{-1}$ & $0.450 \times 10^{-3}$ & 1.834 & 0.470 & 0.754 & 2.975 \\
\hline & 0.70 & 0.5449 & $0.857 \times 10^{-2}$ & $0.212 \times 10^{-3}$ & 2.163 & 0.408 & 0.749 & 2.998 \\
\hline & 0.75 & 0.5313 & $0.487 \times 10^{-2}$ & $0.842 \times 10^{-4}$ & 2.666 & 0.344 & 0.751 & 3.019 \\
\hline & 0.80 & 0.5200 & $0.238 \times 10^{-2}$ & $0.264 \times 10^{-4}$ & 3.504 & 0.277 & 0.752 & 3.038 \\
\hline & 0.85 & 0.5112 & $0.921 \times 10^{-3}$ & $0.574 \times 10^{-5}$ & 5.086 & 0.209 & 0.752 & 3.054 \\
\hline & 0.90 & 0.5050 & $0.234 \times 10^{-3}$ & $0.647 \times 10^{-6}$ & 8.842 & 0.140 & 0.748 & 3.071 \\
\hline \multirow[t]{7}{*}{ II } & 0.0 & 0.4764 & $0.2065 \times 10^{-1}$ & $0.931 \times 10^{-3}$ & 1.487 & 0.464 & 0.681 & 2.647 \\
\hline & 0.1 & 0.3282 & $0.1013 \times 10^{-1}$ & $0.266 \times 10^{-3}$ & 1.929 & 0.401 & 0.744 & 2.685 \\
\hline & 0.2 & 0.2020 & $0.3910 \times 10^{-2}$ & $0.492 \times 10^{-4}$ & 2.599 & 0.282 & 0.807 & 2.797 \\
\hline & 0.25 & 0.1492 & $0.1995 \times 10^{-2}$ & $0.150 \times 10^{-4}$ & 3.239 & 0.221 & 0.861 & 2.749 \\
\hline & 0.3 & 0.1041 & $0.7838 \times 10^{-3}$ & $0.300 \times 10^{-5}$ & 4.854 & 0.149 & 0.993 & 2.409 \\
\hline & 0.35 & 0.0677 & $0.1482 \times 10^{-3}$ & $0.207 \times 10^{-6}$ & 9.285 & 0.095 & 0.986 & 2.512 \\
\hline & 0.38 & 0.0504 & $0.1923 \times 10^{-4}$ & $0.108 \times 10^{-7}$ & 24.06 & 0.063 & 0.825 & 2.924 \\
\hline \multirow[t]{4}{*}{ III } & 1.6 & 0.4253 & $0.4091 \times 10^{-2}$ & $0.198 \times 10^{-3}$ & 1.725 & 0.575 & 0.585 & 4.838 \\
\hline & 1.8 & 0.2839 & $0.6277 \times 10^{-3}$ & $0.936 \times 10^{-5}$ & 2.277 & 0.404 & 0.440 & 7.300 \\
\hline & 1.9 & 0.2212 & $0.9764 \times 10^{-4}$ & $0.503 \times 10^{-6}$ & 2.889 & 0.328 & 0.218 & 13.83 \\
\hline & 1.95 & 0.1928 & $0.1405 \times 10^{-6}$ & $0.937 \times 10^{-7}$ & 3.527 & 0.293 & 0.034 & 28.42 \\
\hline
\end{tabular}

solutions to within a few percent when the distribution and number of model points were varied. For the $\delta=0$ case in model II, our value of $\kappa_{0}$ was checked to be within $2.5 \%$ of the one previous known calculation ${ }^{14}$ of $\kappa_{0}$.

In Figs. 3(a)-3(c) we show the frequency dependence of calculated $\kappa(\omega)$ for the three models at several values of $\delta$. Without exception, our results all confirmed the generic asymptotic frequency dependences given by Eq. (33). It should be noted that in the calculations the finiteelement solutions of the incompressible fluid equations were carried to frequencies just past the crossover frequencies. The nodal points near the wall were adjusted so that there is always a fixed number of them within the viscous boundary layer $l \sim \omega^{-1 / 2}$. These solutions were then matched to the Laplace equation solutions at high frequencies. Smooth matchings were always obtained. In Figs. 4(a)-4(d) we plot the variation of the four asymptotic parameters $\kappa_{0}, C_{1}, \Lambda$, and $\alpha$ for model II as a function of the porosity $\phi$. Since at $\phi_{c}=0.0349$ the throat pinches off, $\alpha$ diverges and the values of $\kappa_{0}, C_{1}$, and $\Lambda$ approach zero as $\phi \rightarrow \phi_{c}$. In Table I all the calculated values of $\kappa_{0}$, $C_{1}, \Lambda$, and $\alpha$, for the three models are listed. It is noted that there is a large variation in their values ranging from $10^{-4}-1$ for $\kappa_{0} / a^{2}, 10^{-7}-10^{-1}$ for $C_{1} / a^{4}, 0.1-1$ for $\Lambda / a$, and $1-20$ for $\alpha$.

\section{SCALING BEHAVIOR OF $\kappa(\omega)$ AND ITS PHYSICAL INTERPRETATION}

A succinct way of displaying all our calculated results is to scale $\kappa(\omega)$ by $\kappa_{0}$ and scale $\omega$ by a characteristic frequency $\omega_{0}=\eta \phi /\left(\rho_{f} \kappa_{0} \alpha\right)$. This would result in a new set of asymptotic parameters for $\widetilde{\kappa}(\widetilde{\omega})$, where $\widetilde{\kappa}=\kappa / \kappa_{0}$, and $\widetilde{\omega}=\omega / \omega_{0}$

$$
\widetilde{\kappa}(\widetilde{\omega})=\left\{\begin{array}{l}
1+i F^{-1} \widetilde{\omega}, \widetilde{\omega} \rightarrow 0, \\
\sqrt{2} F_{2}^{-1} \widetilde{\omega}^{-3 / 2}+i \widetilde{\omega}^{-1}, \quad \widetilde{\omega} \rightarrow \infty .
\end{array}\right.
$$

Here

$$
\begin{aligned}
& F_{1}=\frac{\alpha \kappa_{0}^{2}}{C_{1} \phi} \\
& F_{2}=\left(\frac{\Lambda^{2} \phi}{\alpha \kappa_{0}}\right)^{1 / 2} .
\end{aligned}
$$

This transformation of the variables essentially makes $\kappa_{0}=1$ and $\alpha=1$ so that the low-frequency real part and the high-frequency imaginary part of all the data would 
collapse to the same limiting curves. Comparison with Eq. (33) shows that the dimensionless parameters $F_{1}$ and $F_{2}$ now play the role of $\left(C_{1}^{-1}\right)$ and $\Lambda \alpha$, respectively. If $F_{1}$ and $F_{2}$ should remain constant under microstructural variations, then $\widetilde{\kappa}(\widetilde{\omega})$ would exhibit universal asymptotics. In Figs. 5(a) and 5(b) we plot all the numerical data
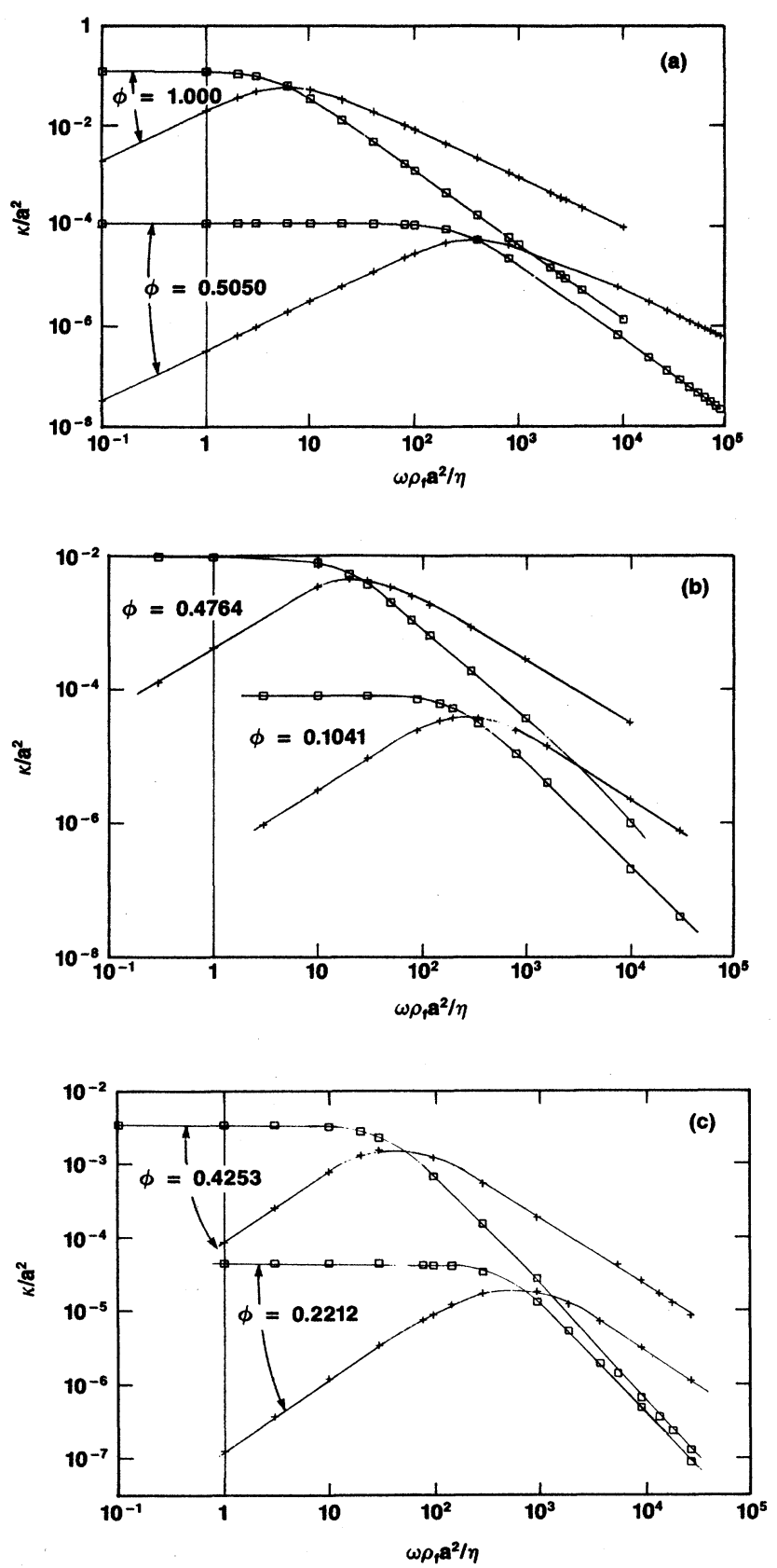

FIG. 3. Numerically calculated permeability as a function of frequency. $\square$-real part, $\dagger$-imaginary part. (a) Model I. The modulation periodicity is taken to be $k=\pi / 5 a$. (b) Model II. (c) Model III. for the three models in scaled variables $\widetilde{\kappa}(\widetilde{\omega})$. It is seen that in spite of drastic porosity and microstructural variations, the scaled data points of all except the two lowest porosity cases of model III, collapse to a single set of curves for the real and imaginary parts not only at high and low frequencies, but also in the transition region as well. That means, first of all, $F_{1}$ and $F_{2}$ are nearly constant for most of our data, which is indeed the case as seen in Table I. In fact, we would like to note here that the near constancy of $F_{2}$ in a variety of sedimentary rocks has been observed by Johnson et al., which is indeed remarkable in view of the diversity in rock's pore geometry. However, the constancy of $F_{1}$ and $F_{2}$ only implies the universality of asymptotic characteristics. If we neglect the two exceptions of model III for the moment, the fact that $\widetilde{\kappa}(\widetilde{\omega})$ also has universal intermediatefrequency crossover behavior indicates that $\kappa(\omega)$ is a scaling function with only two parameters, $\kappa_{0}$ and $\omega_{0}$, which constitute the total geometric content of the dynamic permeability.

The implications of the scaling behavior are that, on the one hand, the dynamic permeability is a poor microstructure probe for porous media since there is only a limited amount of geometric information obtainable form $\kappa(\omega)$; yet on the other hand, it means that there is an underlying simplicity about the dynamic permeability which we would now like to show. Of course, this simplicity should also explain the reason for deviation from the scaling behavior as demonstrated by some of the model III results. The case of the periodic model will first be addressed. The random porous media case is discussed in the next section.

For periodic models, the behavior of the overall system is identical to that of the unit cell. We can therefore focus our attention on the permeability of a single cell. The fluid flow rate $Q$ at any pore cross section may be written as

$$
Q=S U=S[(\kappa / \phi) / \eta](\Delta p / \Delta z)
$$

by invoking the definition of permeability $\kappa$. Here $z$ is the direction of applied pressure gradient and is assumed to be parallel to the cylindrical axis in model I, and to one of the three principal axes in models II and III, $S$ is the cross-sectional area, and the factor $\phi$ normalizes the value of $\kappa$ to just the pore-space volume (instead of the total unit-cell volume). If we write $Q=R^{-1} \Delta p$, then in analogy with electrical systems

$$
R=[\eta \phi /(S \kappa)] \Delta z
$$

can be regarded as flow resistance. For the unit cells in our models, $S$ and $R$ are both functions of $z$. Since the resistors are in series, we can write an effective resistance of the unit cell as

$$
R_{\mathrm{eff}}=\eta \phi L\left[\frac{1}{L} \int_{0}^{L} \frac{d z}{S(z) \kappa(z)}\right],
$$

from which one gets from Eq. (35b)

$$
\kappa_{\mathrm{eff}}=\frac{\eta \phi L}{R_{\mathrm{eff}}\langle S\rangle},
$$


where \langle\rangle denotes averaging. By approximating each small segment $\Delta z$ of the pore space as a straight tube, we can use the asymptotic behavior of $\kappa(\omega)$, Eq. (18), to get the asymptotic parameters $\kappa_{0}, C, \Lambda$, and $\alpha$ of $\kappa_{\text {eff }}$ Expanding in terms of $\omega$ or $\omega^{-1}$ and retaining the leading terms, we get the following geometric interpretation ${ }^{5}$ of the parameters:

$$
\begin{aligned}
\kappa_{0} & =\frac{\phi A}{\left\langle S^{-2}\right\rangle\langle S\rangle}, \\
C_{1} & =\frac{\phi B\left\langle S^{-1}\right\rangle}{\left\langle S^{-2}\right\rangle^{2}\langle S\rangle}, \\
\Lambda & =\frac{\sqrt{2}\left\langle S^{-1}\right\rangle}{C\left\langle S^{-3 / 2}\right\rangle}, \\
\alpha & =\frac{\left\langle S^{-1}\right\rangle\langle S\rangle}{D} .
\end{aligned}
$$

In deriving Eqs. (37a)-(37d) we have assumed that the shape constants $A, B, C$, and $D$ do not vary as a function of $z$, which is valid for model $I$ and approximately valid for models II and III because the shape constants gen-
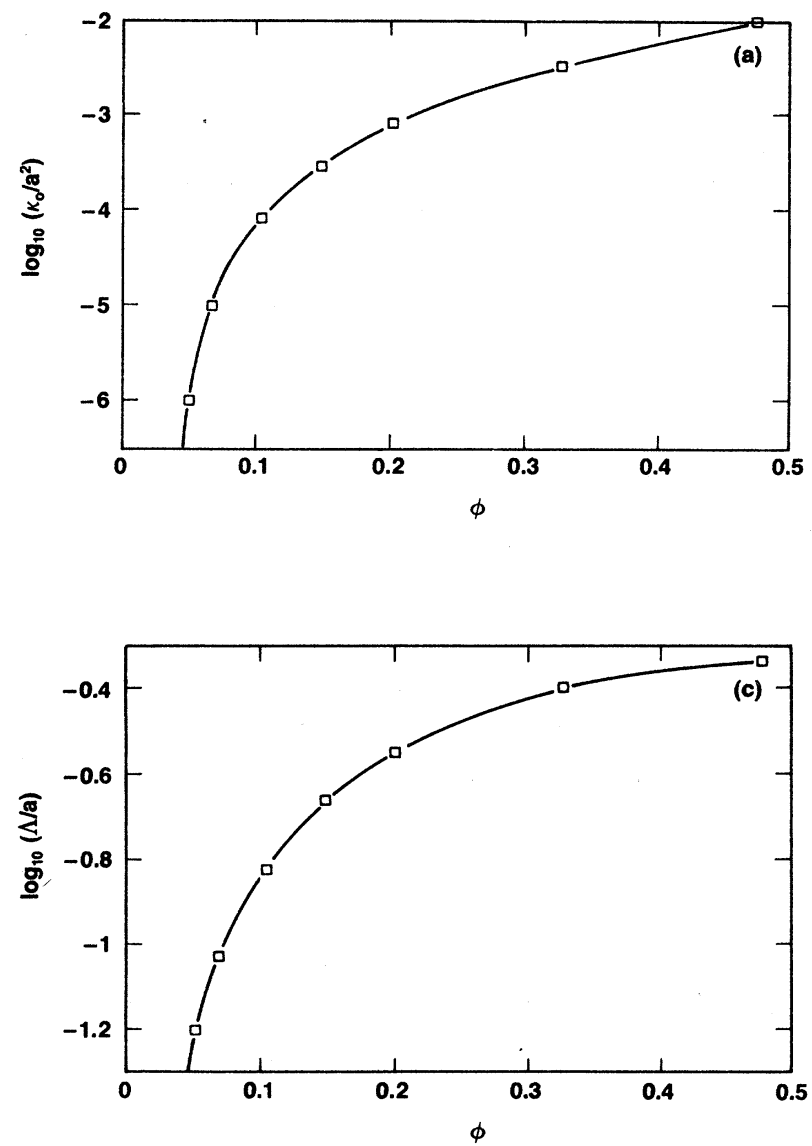

erally do not vary by orders of magnitude. In terms of Eq. (37), $F_{1}$ and $F_{2}$ are given by the following expressions:

$$
\begin{aligned}
& F_{1}=\frac{A^{2}}{B D}, \\
& \left.F_{2}=\left[\int \frac{2 D}{C^{2} A}\right] \frac{\left\langle S^{-1}\right\rangle\left\langle S^{-2}\right\rangle}{\left\langle S^{-3 / 2}\right\rangle^{2}}\right]^{1 / 2} .
\end{aligned}
$$

The important point to note here is that $F_{1}$ is a dimensionless number whose value depends only on the shape constants. For cylindrical tubes, $F_{1}=0.75$. Since we do not expect the shape constants to vary greatly (as $\kappa_{0}, C_{1}$, $\Lambda$, or $\alpha), F_{1}$ is nearly constant. For model I, the calculated value is indeed very close to 0.75 as seen in Table I since the cross section is always circular and therefore $A$, $B, C$, and $D$ have the cylindrical tube values. For models II and III, on the the other hand, the cross-sectional shape of the pores varies as a function of $z$ and can deviate significantly from circular. This results in the deviation of $F_{1}$ from 0.75 . For model II the value ranges from 0.68 to 1 , and for model III the value of $F_{1}$ is mostly
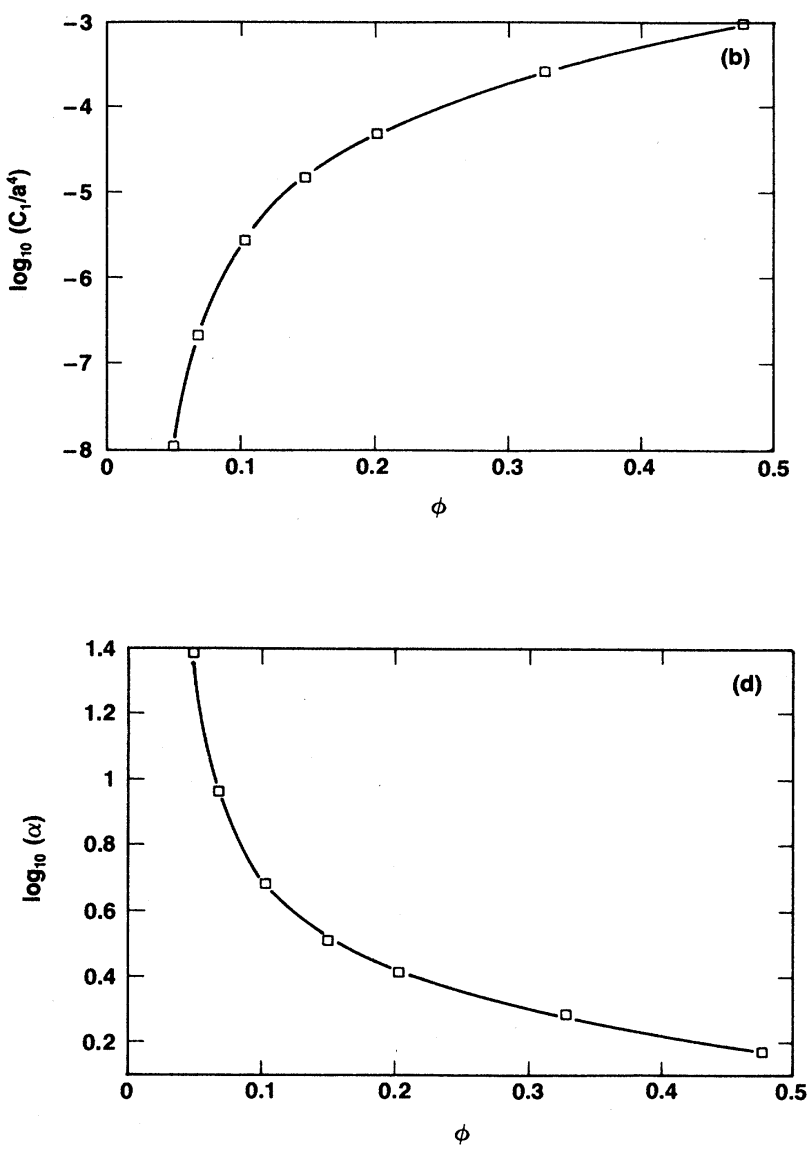

FIG. 4. The asymptotic parameters of model II plotted as a function of porosity $\phi$. (a) $\kappa_{0} / a^{2}$. (b) $C_{1} / a^{4}$. (c) $\Lambda / a$. (d) $\alpha$. At $\phi=0.035$ the pore spaces become disconnected. 
below 0.75 . In contrast to $F_{1}$, the expression for $F_{2}$, Eq. (38b), is noted to contain the averages of the crosssectional areas as well as the shape constants. However, all the averages involve $S$ raised to a negative power. This is significant because if $S$ were constant, then $F_{2}=\left(2 D / C^{2} A\right)^{1 / 2}=\sqrt{8} \simeq 2.83$ for cylindrical tubes, but if $S$ has very large variations, then $\left\langle S^{-n}\right\rangle \simeq S_{\mathrm{th}}^{-n}$, where $S_{\text {th }}$ is the area of the throat, i.e., the region of the smallest cross section, and $F_{2}$ would again be close to $\sqrt{8}$. Therefore, from interpolation between these two extreme limits we conclude that $F_{2}$ is also nearly constant. Similar reasoning shows that $\Lambda$, Eq. (37c), should be interpreted as a volume-to-surface ratio for the throat region instead as an average for the entire pore space. The above line of argument clearly demonstrates that while the magnitudes of $\kappa_{0}, C_{1}$, and $\alpha$ are determined by more than just the throat area, yet in the scaled variables $\widetilde{\kappa}$ and $\widetilde{\omega}$, the func-
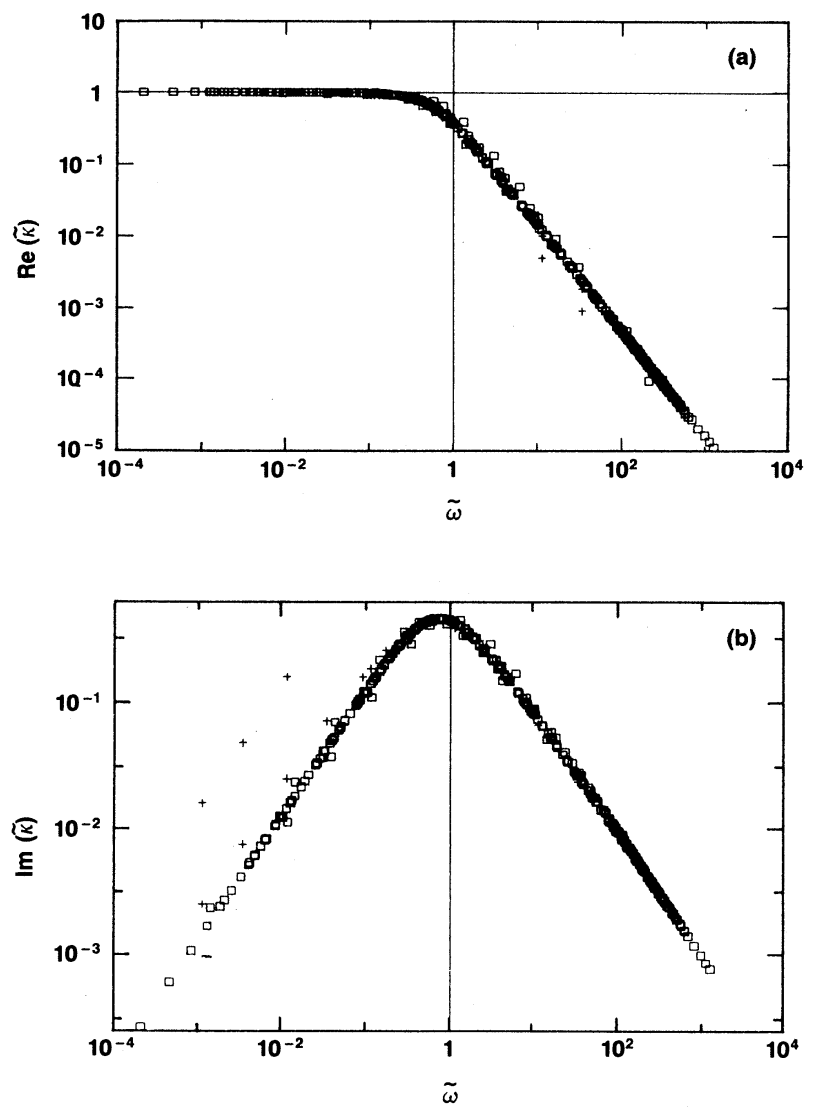

FIG. 5. (a) The real part, and (b) the imaginary part, of the scaled dynamic permeability $\widetilde{\kappa}(\widetilde{\omega})$. The model parameters from which the data are generated can be found in Table I. All except the two lowest-porosity cases of model III are plotted in the open square $(\square)$ symbol. The two lowest-porosity cases of model III are shown in the cross $(+)$ symbol. It is seen that all except the two cases of model III lie on the same curve. The two exceptions both have small pore throats defined by sharp knife edges. The physical reasons for the scaling behavior and the deviation from it are discussed in the text. tion $\widetilde{\kappa}(\widetilde{\omega})$ is dominated by the throat. Therefore, if the throat region is straight-tube-like, then $\widetilde{\kappa}(\widetilde{\omega})$ should exhibit scaling behavior in the intermediate frequency range as well. It follows that the criterion for the validity of the scaling behavior is for the throat region of the pore space to be straight-tube-like, defined more precisely as $d S / d z \simeq 0$, where $z$ is the normal distance away from the throat. This simple geometrical interpretation of the scaling behavior is noted to be self-consistent in the sense that the criterion of $d S / d z \simeq 0$ is a requirement for $\mathrm{Eq}$. (36a) to be a reasonable approximation in the first place. A situation that would clearly violate this condition is where $S(z)$ varies extremely fast as one moves away from the throat, e.g., two pores connected by a small hole made in a thin sheet. In fact, for model III the throat area has $d S / d z \neq 0$, and we can clearly see the deviations form the scaling behavior for the two lowest-porosity cases where the throat size becomes small and the $F_{1}, F_{2}$ values deviate significantly from 0.75 and $\sqrt{8}$.

To summarize, the main conclusion of this section is that the transformation of the variables $\widetilde{\kappa}=\kappa / \kappa_{0}$ and $\widetilde{\omega}=\omega / \omega_{0}$ results in a function $\widetilde{\kappa}(\widetilde{\omega})$ which is throat dominated. If the throat region of the pore space satisfies the criterion of $d S / d z \simeq 0$, then scaling behavior is expected to hold and $\kappa_{0}$ and $\omega_{0}$ are the only two pieces of geometric information obtainable from the unscaled $\kappa(\omega)$ function. However, if $d S / d z$ is finite and large at the throat, then scaling breaks down and the deviation of $\widetilde{\kappa}(\widetilde{\omega})$ from the cylindrical-tube behavior is an indication of how acute the knife edge is that defines the throat.

\section{RANDOM MEDIA SIMULATION AND CRITICAL-PATH INTERPRETATION OF RESULTS}

The knowledge about the unit-cell behavior in the periodic porous models provides a basis for simulating the behavior of random porous media. The question we would like to answer is the following: Can the scaling behavior, if it exists for each unit, be preserved in a random system comprising of a network of permeable elements, each characterized by two parameters $\kappa_{0}$ and $\omega_{0}$ randomly sampled from their respective distribution functions? To this end we have considered a simple-cubic lattice made from random permeable bonds. By using exponential, flat, and log-normal distributions for the parameters $\kappa_{0}$ and $\omega_{0}$, and then deducing the value of $\kappa(\omega)$ for each bond $i j$ (between nearest-neighbor nodes $i$ and $j$ ) by

$$
\kappa_{i j}(\omega)=\kappa_{0}(i j) \widetilde{\kappa}\left(\omega / \omega_{0}(i j)\right)
$$

[where the analytic cylindrical-tube solution is used for $\widetilde{\kappa}(\widetilde{\omega})$ ], we have solved essentially the Kirchhoff equations on $9 \times 9 \times 9$ lattices. The results are shown in Fig. 6. The scaling is generally excellent except for the small differences in the low-frequency imaginary part and the high-frequency real part. These differences are explainable in terms of the critical-path argument as shown below. Compared with the cylindrical-tube solution, it is noted that the random porous media generally have a somewhat broader transition region for $\widetilde{\kappa}(\widetilde{\omega})$.

What can be the reason for the scaling behavior in random systems? Of course, if the distributions of the pa- 


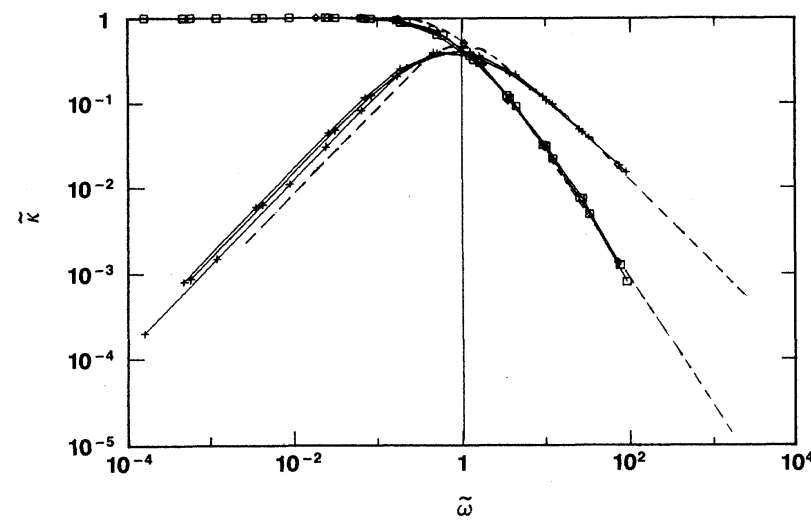

FIG. 6. Scaled dynamic permeabilities of $9 \times 9 \times 9$ networks of random permeable bonds plotted as a function of scaled frequency. Data for the real part are shown as $\square$, the imaginary part as + . The three cases represent (1) uniform distributions of $\kappa_{0}(i j)$ and $\omega_{0}(i j)$ in the interval $(0,[1],(2)$ exponential distributions $\exp (-m x) / m$ with $m=1$ for $\kappa_{0}(i j)$ and $m=0.5$ for $\omega_{0}(i j)$, and (3) log-normal distributions $\exp \left[-(\ln x-\mu)^{2} / 2 \sigma^{2}\right] /$ $\sigma x \sqrt{2 \pi}$ with $\mu=3, \sigma=0.5$ for $\kappa_{0}(i j)$ and $\mu=0.3, \sigma=1.5$ for $\omega_{0}(i j)$. The tube solution is shown by the dashed lines. The slight differences in the low-frequency imaginary parts and the high-frequency real parts reflect the differences in the constants $F_{1}$ and $F_{2}$ as explained in the text.

rameters are sharply peaked, one would expect the overall system behavior to be similar to that of the individual elements. On the other hand, if the distributions are broad, we expect the critical path to dominate. That is, if we regard two nearest-neighbor nodes $i j$ of our network to be connected if their $\kappa_{0}(i j)>\kappa_{b}$, then the overall permeability of the system is expected to be dominated by $\kappa_{b}=\kappa_{c}$ at which the connected nodes form an infinite network. This argument, originally proposed by Ambegaokar, Halperin, and Langer ${ }^{15}$ for the explanation of Mott's $\exp \left[-\left(T_{0} / T\right)^{1 / 4}\right]$ temperature dependence of the hopping conductivity in disordered semiconductors, was subsequently employed to explain the temperature dependence of the tunneling conductivity in a variety of inhomogeneous systems, ${ }^{16}$ as well as the permeabilityformation factor correlation in sedimentary rocks. ${ }^{1}$ By using this picture it becomes clear that the static permeability is dominated by a few key linkages in the criticalpath network. Since, by definition, these key linkages have to be $>\kappa_{c}(\omega=0)$ but yet not $\gg \kappa_{c}$ (since otherwise the critical path would be formed at a $\kappa_{c}^{\prime}>\kappa_{c}$ ), it follows that they must have a relatively narrow distribution in their $\kappa_{0}$ values. At high frequencies, a similar argument would imply a narrow distribution of the $\alpha$ values for a different set of the critical linkages. The $\kappa_{c}, \alpha_{c}$ would therefore be the two scaling parameters for the system, and the magnitude of $\kappa(\omega)$ is expected to follow the scaling behavior. For the phase, the imaginary part of $\kappa(\omega)$ at low frequencies is always a small fraction of the static permeability $\kappa_{0}$. Therefore, either serial or parallel connection of the critical $\kappa_{i j}(\omega)$ 's would result in the arithmetic addition of their imaginary parts. ${ }^{17}$ That means the $C_{1}$ coefficient of the overall system is expressible as the average of the individual $C_{1}$ 's:

$$
\left\langle C_{1} / \phi\right\rangle=\left\langle\frac{\alpha \kappa_{0}^{2}(i j)}{F_{1} \phi^{2}}\right\rangle \simeq \frac{\kappa_{c}^{2}}{F_{1} \phi^{2}}\langle\alpha\rangle,
$$

where we have taken $\kappa_{0}^{2}(i j) / \phi^{2} \simeq \kappa_{c}^{2} / \phi^{2}$ from the criticalpath argument and noted that $F_{1}$ is a constant for every permeable bond. If now we define

$$
F_{1}^{\prime}=\frac{\alpha_{c} \kappa_{c}^{2}}{\left\langle C_{1}\right\rangle \phi}
$$

as a new constant for the system, we get the relation

$$
F_{1}^{\prime}=\frac{\alpha_{c}}{\langle\alpha\rangle} F_{1}
$$

by treating $\phi$ as a constant. That is, to the extent that $\alpha_{c}$ deviates from $\langle\alpha\rangle$, the constant $F_{1}$ of the random system would depart from the $F_{1}$ value of the individual elements. At high frequencies the real part of $\kappa(\omega)$ plays the similar role as the imaginary part at low frequencies. Therefore,

$$
\langle\Lambda\rangle=F_{2} \alpha_{c}^{1 / 2}\left\langle\left(\frac{\kappa_{0}}{\phi}\right)^{1 / 2}\right\rangle,
$$

where it is now the $\alpha$ that is determined by the critical path, and $\kappa^{-1 / 2}$ is averaged. By defining

$$
F_{2}^{\prime}=\left(\frac{\langle\Lambda\rangle^{2} \phi}{\alpha_{c} \kappa_{c}}\right)^{1 / 2},
$$

we get

$$
F_{2}^{\prime}=\left(\frac{\left\langle\kappa_{0}^{1 / 2}\right\rangle}{\kappa_{c}^{1 / 2}}\right) F_{2},
$$

where $\phi$ is again taken to be constant.

The above discussion shows that the random systems should exhibit scaling behavior ${ }^{18}$ but with modified values for $F_{1}$ and $F_{2}$. That means samples with the same distributions of $\omega_{0}(i j)$ and $\kappa_{0}(i j)$ should have the same $\widetilde{\kappa}(\widetilde{\omega})$, but samples with different distributions of $\omega_{0}(i j)$ and $\kappa_{0}(i j)$ can differ slightly in their $F_{1}$ and $F_{2}$ values as seen in Fig. 6. This generalization has immediate implications for the explanation of observed correlations in sedimentary rocks. For example, the product of the static permeability and the formation factor can be expressed as

$$
\frac{\kappa_{c} \alpha_{c}}{\phi}=\frac{\langle\Lambda\rangle^{2}}{F_{2}^{\prime 2}}
$$

from Eq. (40b). By noting from Eq. (37c) that $\Lambda$ is expressible in terms of $\left\langle S^{-n}\right\rangle$, we see that $\Lambda$ is a volumeto-surface ratio for the throat region, in agreement with the conclusion of $\mathrm{Katz}$ and Thompson. ${ }^{1}$ Similarly, the noted near constancy of $F_{2}$ in a variety of rocks ${ }^{3}$ may be interpreted as an indication that the condition for the validity of the scaling behavior is indeed satisfied in a plurality of naturally occurring porous materials. 
In order to explicitly verify our scaling prediction, experimental measurements of $\kappa(\omega)$ have been performed on fused-glass beads and crushed-glass samples. ${ }^{6}$ Excellent agreement with the theory was found. We therefore conclude that the scaling behavior may be regarded as generally valid, with the static permeability and the characteristic rollover frequency as the two geometric parameters characterizing the medium. Provided the values of the two constants $F_{1}$ and $F_{2}$ are known, the knowledge of any two of the four asymptotic parameters would therefore be sufficient to deduce the values of the other two. That means if the tortuosity is determined from the dc electrical formation factor and $\Lambda$ is estimated from volume-to-surface ratio, then $\kappa_{0}$ and $C_{1}$ may be inferred with reasonable accuracy.

\section{CONCLUDING REMARKS}

As we have made clear in Sec. II, a crucial assumption that underlies our results is that the sound wavelength $\lambda \sim L=\tau c_{0} \gg a$. At extremely high frequencies, this assumption breaks down and one has to take into account the compressibility of the fluid. Even in the simple tube geometry, the problem becomes nontrivial, and its solution will be reported elsewhere. Another possible complication is the large Reynold's number that can result from large pressure gradients. One would then be forced to employ the full Navier-Stokes equation in the calculation of $\kappa(\omega)$, and a whole new set of characteristics is expected. However, in our view the most intriguing topic for future research lies in the regime where the pore size becomes comparable to the size of fluid molecules so that the fluid can no longer be treated as a continuum. Due to the widespread use of molecular sieves (such as zeolites) in chemical industries, such porous materials are no longer just laboratory curiosities. However, the theoretical study of their permeability behavior is expected to require the development of new theoretical techniques, which is a problem presently under further consideration.

\section{ACKNOWLEDGMENTS}

We wish to thank J. P. Stokes, E. Charlaix, and D. L. Johnson for helpful discussions during the course of this work.
*Permanent address: Department of Physics, Shanghai Teacher's University, Shanghai, People's Republic of China.

${ }^{1}$ A. J. Katz and A. H. Thompson, Phys. Rev. B 34, 8179 (1986).

${ }^{2}$ A. H. Thompson, A. J. Katz, and C. E. Krohn, Adv. Phys. 36, 625 (1987).

${ }^{3}$ D. L. Johnson, J. Koplik, and L. M. Schwartz, Phys. Rev. Lett. 57, 2564 (1986).

${ }^{4}$ D. L. Johnson, J. Koplik, and R. Dashen, J. Fluid Mech. 176, 379 (1987).

${ }^{5}$ P. Sheng and M. Y. Zhou, Phys. Rev. Lett. 61, 1591 (1988).

${ }^{6}$ E. Charlaix, A. Kushnick, and J. P. Stokes, Phys. Rev. Lett. 61, 1595 (1988).

${ }^{7}$ R. Burridge and J. B. Keller, J. Acoust. Soc. Am. 70, 1140 (1981). Our treatment of the $\epsilon$ scaling differs from the reference above in that we treat $\omega$ as an external parameter, and all the scaling units are taken from intrinsic material constants of the medium.

${ }^{8}$ M. A. Biot, J. Acoust. Soc. Am. 34, 1254 (1962).

${ }^{9}$ A. Bedford, R. D. Costley, and M. Stern, J. Acoust. Soc. Am. 76, 1804 (1984).
${ }^{10}$ A. N. Norris, J. Wave-Material Interact. 1, 380 (1986).

${ }^{11}$ Written by Bengt Fornberg. We wish to thank him for supplying us with this code.

${ }^{12}$ Lord Rayleigh (J. W. Strutt), Philos. Mag. 34, 481 (1892).

${ }^{13}$ R. J. S. Brown, Geophysics 45, 1269 (1980).

${ }^{14}$ A. S. Sangani and A. Acrivos, J. Multiphase Flow 8, 343 (1982).

${ }^{15}$ V. Ambegaokar, B. I. Halperin, and J. S. Langer, Phys. Rev. B 4, 2612 (1971).

${ }^{16}$ P. Sheng and J. Klafter, Phys. Rev. B 27, 2583 (1983); J. Klafter and P. Sheng, J. Phys. C 17, L93 (1984).

${ }^{17}$ This fact is clear for the serial connection case. For the parallel connection case, if the real part of the permeability for the elements comprising the critical-path network has a narrow distribution centered around $\kappa_{c}$, then the stated result is again true provided the imaginary parts are small compared to $\kappa_{c}$.

${ }^{18} \mathrm{~A}$ special case of our random networks where the permeable elements are random-diameter tubes (so that instead of two independent parameters $\kappa_{0}$ and $\omega_{0}$, there is only one) was in fact demonstrated in Ref. 4 to exhibit tubelike behavior. 